\title{
Development and Evaluation of a Novel Radiotracer 125I-rlL-27 to Monitor Allotransplantation Rejection by Specifically Targeting IL-27Ra
}

Feng Gao ( $\sim$ rggaofeng@sdu.edu.cn )

Shandong University Cheeloo College of Medicine

Shanshan Zhao

Shandong University Cheeloo College of Medicine

Guihua Hou

Shandong University Cheeloo College of Medicine

Original research

Keywords: IL-27, allograft rejection, radionuclide imaging

Posted Date: January 15th, 2021

DOl: https://doi.org/10.21203/rs.3.rs-145989/v1

License: (c) (i) This work is licensed under a Creative Commons Attribution 4.0 International License.

Read Full License 


\section{Abstract}

Non-invasive monitoring of allograft rejection is benefit for the prognosis of patients with organ transplantation. Recently, IL-27/IL-27Ra was proved in close relation with inflammatory diseases, and ${ }^{125}$ I-anti-IL-27Ra mAb our group developed demonstrated high accumulation in rejecting allograft. However, antibody imaging has limitation in the imaging background due to its large molecule weight. Therefore, we developed a novel radio tracer (lodine-125 labeled recombinant IL-27) to evaluate the advantage on the targeting and imaging of allograft rejection. In vitro specific binding of ${ }^{125} \mathrm{I}$-rlL-27 was determined by saturation and competitive assay. Blood clearance, biodistribution, autoradio-imaging and IL-27Ra expression were studied on day 10 post transplantation (top period of allorejection). Our results indicated that ${ }^{125} \mathrm{I}$-rIL-27 could bind with IL-27Ra specifically and selectively in vitro. Blood clearance assay demonstrated a fast blood clearance with $13.20 \mu \mathrm{l} / \mathrm{h}$ of ${ }^{125} \mathrm{I}-\mathrm{rlL}-27$ staying in blood after $24 \mathrm{~h}$. Whole-body phosphor-autoradiography and biodistribution assay indicated that higher specific uptake of ${ }^{125} \mathrm{I}$-rIL-27 and clear radio-image in allograft than syngraft at $24 \mathrm{~h}$, while similar result was obtained at $48 \mathrm{~h}$ in group of ${ }^{125} \mathrm{I}$-anti-IL-27Ra mAb injection. Meanwhile, higher IL-27Ra expression was found in allograft by the western blot study. The activity accumulation of ${ }^{125}$ I-rIL-27 was highly correlated with IL27Ra expression on allograft. In conclusion, ${ }^{125} \mathrm{I}$-rlL-27 could be a promising probe to acutely monitor the allograft rejection with high specific binding towards IL-27Ra on allograft and low imaging background.

\section{Introduction}

Solid organ allotransplantation has been the most effective therapeutic strategy for the patient with endstage organ failure[1]. However, the occurrence of acute rejection is strongly related with the allograft loss and the poor prognosis[2]. Therefore, early detection of acute rejection with non-invasive method could greatly benefit for the prognosis after organ transplantation[3].

Recently, IL-27Ra (IL-27 receptor a), along with its ligand IL-27, is evidenced to trigger the immune response, including cancer, Abdominal aortic aneurysm, Sjögren's syndrome, virus infection and transplantation[4][5][6][7][8]. IL-27Ra is the specific subunit of IL-27 receptor and restricted mainly to lymphocytes and monocytes[9]. IL-27 pathway has been proved to inhibit tumor growth by enhanced T cells response and decreased Treg cells (T regulator cells) proportion[10]. Besides, IL-27 displayed a proinflammatory effect by enhancing the IL-1 $\beta$ secretion from monocytes and macrophages[11]. Moreover, IL-27 could also promote NK cell function by secreting more IFN- $\gamma$ during influenza infection[12]. All these suggested that IL-27 could activate IL-27Ra and enhance pro-inflammation response.

Acute allograft rejection is a severe pro-inflammation participated by T cells and macrophage[13] and IL27 has been proved in close relation with allograft rejection[7][14]. IL-27Ra (IL-27 receptor a) expression on T cells exacerbated GVHD by enhancing effector function of Th1 cells (T helper 1 cells) and inhibiting Th2 and Treg cell subsets[7], while IL-27Ra was found apparently up-regulated on alloreactive splenetic $\mathrm{CD}^{+}{ }^{+} \mathrm{T}$ cells, $\mathrm{T}$ cell and macrophage when acute rejection happened[15][16][17]. In our previous study with 
allografted mice model, we found a great amount of IL-27Ra positive T cells and macrophage infiltrated in rejecting allograft and iodine-125 labeled anti-IL-27Ra mAb could obviously accumulate in allograft non-invasively when rejection occurred[18].

Target tissue could be diagnosed precisely and non-invasively by nuclear molecular imaging with a specific probe, which is much more favorable than histopathological biopsies and traditional imaging examination[19][20][21][22][23]. Although histopathological biopsies was the "gold-standard" of acute graft rejection, it still was an invasive examine and may induce complication including pain, bleeding and death[24][25]. The non-invasive examines such like MRI and ultrasound reflected the decrease graft function and were limited in targeting allograft[21][23]. Targeted molecular imaging has advantages in tracking specific cells and monitoring the function of the target organ with the probes which have the detection signals [26][27][28]. Among them, radionuclide imaging is a non-invasive method by which the disease could be diagnosed effectively and timely, and the therapeutic effect could be monitored with the help of radio-probe. Radionuclide imaging with radiolabeled macromolecular such as protein, antibody and so on usually had the disadvantages in the long time to reach the target tissue and the high background, resulting in the poor imaging quality. However, small molecular could accumulate in the target tissue quickly, and thus make a better imaging. Therefore, radio-probe with small size is the much more promising radio tracer in radionuclide imaging compared with full length antibody. Radio-labeled cytokine has been applied to track targeted immune cells due to the high-contrast imaging, fast clearance, low background and the weak inflammation response. [29][30][31]. Hartimath et al developed [18F]FB-IL-2 to monitor cancer therapy-induced activated T lymphocyte infiltration in tumor[32]. Di Gialleonardo et al demonstrated that ${ }^{18} \mathrm{~F}$-FB-IL-2 could trace IL-2 receptor-positive cells[33]. Ding et al suggested [ $\left.{ }^{124} \mathrm{I}\right] \mathrm{I}-\mathrm{F} 8 \mathrm{-IL}-10$ could accumulate in arthritic joints in rheumatoid arthritis patient. Meanwhile, fast clearance of [ $\left.{ }^{124} \mathrm{I}\right] \mathrm{I}-\mathrm{F} 8-\mathrm{IL} 10$ and [ $\left.{ }^{131} \mathrm{I}\right] \mathrm{I}$-F8-IL-10 in non-specific target tissues was found within the a span of 24h[34]. Accordingly, imaging with radiolabeled cytokine had advantage in specific recognition of target tissue with low background, and could be a promising strategy for allorejection detection.

In this study, we prepared a novel radio-probe $\left({ }^{125}\right.$ l labeled recombinant IL-27, ${ }^{125} \mathrm{I}$-rlL-27) to specifically target IL-27Ra, and evaluated its possibility for the potential application in acute allograft rejection monitoring.

\section{Materials And Methods}

\subsection{Chemicals, reagents, and equipment}

IL-27Ra mAb was obtained from R\&D system (Minnesota, USA). Recombination IL-27 (rlL-27) was purchased from peprotech (New Jersey, USA). $\mathrm{Na}^{125}$ I was provided by China Institute of Atomic Energy (Beijing, China). SephadexG-25M PD10 column was purchased from GE Healthcare (Pennsylvania, USA). RPMI 1640 medium was get from Biological Industries (Kibbutz Beit Haemek, Israel). HEPES buffer and Red Blood Cell lysis buffer were got from Solarbio (China, Beijing). SDS loading buffer, antibody dilution buffer and blocking buffer were obtained from Beyotime (Shanghai, China). PBS TBST buffer, H\&E 
staining and immunofluorescence (IF) staining regent were purchased from Servicebio (Wuhan, China). GAPDH solution was obtained from Bioss (Beijing, China) and Bioworld (Illinois, USA). HRP-labeled Goat Anti-Rat IgG solution and HRP-labeled Goat Anti-Rabbit IgG solution was get from EpiZyme (Shanghai, China). ECL substrate was purchased from Merck Millipore (Darmstadt, Germany).

The radioactive counts were measured by Gamma Counter from Capintec Inc (USA). The phosphorautoradiography imaging was captured and analyzed by Cyclone Plus Scanner (PerkinElmer, Life Sciences, USA). The membrane was scanned by Tanon 5200 imaging system scanner (Tanno, Shanghai, Beijing).

\subsection{Radiochemistry}

\subsubsection{Preparation of the Radio-probe}

The preparation of ${ }^{125}$ I labeled probe was performed according to the reference[35]. Briefly, 0.05M PB solution $(100 \mu \mathrm{L})$, IL-27Ra mAb $(12 \mu \mathrm{g})$ or rIL-27 $(8 \mu \mathrm{g})$ and $\mathrm{Na}^{125}$ I $(11.9 \mathrm{MBq})$ was mix in the tube with lodogen. Then the mixture was added into the SephadexG-25M PD10 column, following by the elution with $0.01 \mathrm{M}$ PB solution. The eluent was collected in tube $(0.5 \mathrm{ml}$ for each tube) and the radioactive count of $10 \mu \mathrm{L}$ eluent from each tube was measured by Gamma Counter.

The radiochemical purity were detected following the protocol. Briefly, $2 \mu \mathrm{L}$ of the radio-probe was added into the filter paper ( $2 \mathrm{~cm}$ to the bottom). Then bottom of the paper was immersed in the solution of $0.9 \%$ saline and methanol $(1: 2, \mathrm{v} / \mathrm{v})$. After $40 \mathrm{~min}$, the paper was cut into slice $(1 \mathrm{~cm})$ and radioactive count was measured by Gamma Counter.

\subsubsection{In vitro stability study}

Radio-probe $(12.5 \mu \mathrm{L})$ was dissolved in saline $(100 \mu \mathrm{L})$ or mouse serum $(100 \mu \mathrm{L})$, and the mixture was kept at $37^{\circ} \mathrm{C}$ for a period of time. At $1,12,24 \mathrm{~h}, 2 \mu \mathrm{L}$ of the sample was taken out and analyzed so as to observe the change of radiochemical purity

\subsubsection{Determination of lipophilicity}

${ }^{125} \mathrm{I}-\mathrm{rIL}-27\left(0.2 \mu \mathrm{L}, 4.08 \times 10^{-4} \mathrm{MBq}\right)$ was diluted in $1 \mathrm{M}$ HEPES buffer $(500 \mu \mathrm{L})$ and mixed with n-octanol $(500 \mu \mathrm{L})$ for $30 \mathrm{~min}$, following by the centrifugation for $10 \mathrm{~min}$ with $14000 \times \mathrm{g}$. Subsequently, Aliquots of $\mathrm{n}-$ octanol and water phases $(400 \mu \mathrm{L})$ was taken out and then centrifuged again. Finally, the radioactive count of each phase $(100 \mu \mathrm{L})$ was measured by Gamma Counter and the octanol/water partition coefficient ( $\left.\log \mathrm{D}_{\mathrm{o} / \mathrm{w}}\right)$ was calculated.

\subsection{Cell Assays}

Cell assays were performed using spleen cells isolated from the mouse model on day 10 post transplantation. Briefly, spleen of mouse model was isolated and pressed on mesh 200 . Then, cells were 
treated with Red Blood Cell lysis buffer, washed with PBS and final suspended in RPMI 1640 medium. Cells were cultured in 48-well plates for $2 \mathrm{~h}$ with each well $1 \times 10^{6}$ cells in $200 \mu \mathrm{L}$ RPMI 1640 medium, and used for further studies after attachment.

\subsubsection{Competition study}

For competition binding assay, non-labeled anti-IL-27Ra mAb $(0$ to $71.4 \mu \mathrm{M})$ was incubated with alloreactive spleen cells for $1 \mathrm{~h}$ before $147.09 \mathrm{nM}{ }^{125} \mathrm{I}$-rlL-27 was added. Wash the cells with cold PBS buffer twice and discard the supernatant. The activity bound in the cells was measured by Gamma Counter. $B / B_{0}$ was described as the ratio of radioactive counts with non-labeled anti-IL-27Ra mAb to the radioactive counts without non-labeled anti-IL-27Ra mAb. The inhibition constant ( $K_{i}$ value) was calculated in GraphPad Prism software.

\subsubsection{Saturation study}

${ }^{125}$ I-rlL-27 (3.68 to $147.09 \mathrm{nM}$ ) was incubated with spleen cells for $2 \mathrm{~h}$ at $37^{\circ} \mathrm{C}$ to obtain the total activity binding of ${ }^{125} \mathrm{I}$-rlL-27.In order to test the non-specific binding, cells were pre-treated with $10.46 \mu \mathrm{M}$ nonlabeled rIL-27 for 1 hour.

After incubation with ${ }^{125}$-rlL-27, cells were washed with cold PBS buffer twice and radioactive counts were measured in Gamma Counter. The maximum binding capacity $\left(\mathrm{B}_{\max }\right)$ and dissociation constant $\left(K_{d}\right)$ were calculated in GraphPad Prism software. The specific binding was the value of total binding minus non-specific binding.

\subsection{Small animal in vivo experiments}

All animal experiments were performed in agreement with the ARRIVE guidelines. The protocol was approved by the Animal Care and Use Committee of the University with the corresponding ethical approval code (LL-201602040, 2016-2022). Female BALB/c mice (H-2d) and C57BL/6 mice (H-2b) were purchased from Vital River Laboratory Animal Technology (Beijing, China) and housed under standard conditions with free access to water and standard food.

\subsubsection{Animal models}

To establish the skin transplantation model, C57BL/ 6 mice and BALB/c mice were employed as the skin graft donor of allogeneic and syngeneic transplantation, respectively. BALB/c mice were the recipients. Briefly, surgery was performed under anesthesia with $0.6 \%$ pentobarbital sodium $(0.1 \mathrm{~mL} / 10 \mathrm{~g}$ body weight) in sterility condition. The mucous membrane and blood vessel of graft was removed and then cut the graft into circle with $1 \mathrm{~cm}$ in diameter. Then, remove the skin of recipients in right shoulder and transfer the graft to recipients. Finally, petrolatum gauze was put on the graft and covered with bandage. Acute rejection occurred on day 7 post transplantation when removing the bandage with escharotics area over $50 \%$. 


\subsubsection{Blood clearance assay}

At $1 \mathrm{~h}, 2 \mathrm{~h}, 6 \mathrm{~h}, 12 \mathrm{~h}$ and $24 \mathrm{~h}$ post injection of radio-probe, mice were anaesthetized with $0.6 \%$ pentobarbital sodium solution. Then $5 \mu \mathrm{L}$ blood was collected from the tail vein. The activity of radio-probe stayed in the blood was counted by Gamma Counter. Each mouse was weighted and the concentration of radioprobe in blood $\left(\mathrm{ng} / \mathrm{\mu L}\right.$ ) was calculated using $78 \mathrm{~mL} / \mathrm{kg}$ as blood factor. AUCs (Area Under Curves) of ${ }^{125}$ -

rlL-27 in 24h and ${ }^{125}$-anti-IL-27Ra mAb in 48h were obtained using GraphPad Prism software. Blood clearance $(\mathrm{CL}, \mu \mathrm{l} / \mathrm{h})$ was calculated as dose/AUC with the study referred[36].

\subsubsection{Dynamic phosphor-autoradiography}

Mice were divided into allo-group, syn-group and blocking group ( $\mathrm{n}=5$ for each group) according allogeneic, syngeneic transplantation and allogeneic transplantation model with specific antibody blocking. After fed with $3 \% \mathrm{Nal}$ solution for $24 \mathrm{~h}, 60 \mu \mathrm{g}$ non-labeled IL-27Ra mAb was injected to the blocking group. One hour later, all mice were injected with ${ }^{125} \mathrm{I}$-rlL-27 $(0.37 \mathrm{MBq})$ and ${ }^{125} \mathrm{I}$-anti-IL-27Ra $\mathrm{mAb}(0.37 \mathrm{MBq})$ on day 9 post transplantation, respectively. Mice were anesthetized and scanned by Cyclone Plus Scanner. Regions of interest (ROIs) were quantified using the OptiQuant Image Analysis Software and presented as Digital Light Units per square millimeter (DLU/mm²).

\subsubsection{Ex vivo Biodistribution}

Three groups of mice (allo-group, syn-group, and blocking group, $n=3$ for each group) were sacrificed with at $24 \mathrm{~h}$ after intravenous injection of radio-probe $(0.08 \mathrm{MBq}$ in $200 \mu \mathrm{L}$ of $0.01 \mathrm{M} \mathrm{PB})$. Organs or tissues of interest including blood, liver, lung, kidney, spleen, control skin and graft were excised and weighed. The activity was measured by Gamma counter and the uptake of radio-probe was expressed as the percentage of injected dose per gram (\%ID/g). T/NT (Target/non-Target) ratio was calculated by dividing the \%ID/g of the target graft to that of the control skin (opposite site), while T/B (Target/Blood) was $\% \mathrm{ID} / \mathrm{g}$ of the target graft to that of the blood.

\subsubsection{H\&E (hematoxylin and eosin) staining and Immunofluorescence staining}

On day 10 post transplantation, grafts were collected and histological sections were prepared. H\&E staining and immunofluorescence (IF) staining were performed following the protocols of staining kit. The image was obtained under the optical microscope. Briefly, in H\&E staining, the sections were covered haematoxylin for $5 \mathrm{~min}$. After $1 \%$ acid ethanol regent for 5 second, sections were covered with blue promoting solution for 5 seconds. Next, the sections were covered with eosin solution for 10 minutes. Between every step, the distilled water was used to wash out the excess buffer. In IF staining, the sections were treated with EDTA antigen repair buffer $(\mathrm{pH} 9.0)$ and blocked with BSA for 30min. Then, anti-IL-27Ra Ab was diluted in PBS (1:200) and added on the section in $4^{\circ} \mathrm{C}$, overnight. The sections were washed with PBS and covered with second antibody for 1 hour. Later, wash the sections with PBS and add the FITC regent (Green) on the sections. Next, the sections were washed with TBST and covered with tissue autofluorescence quencher regent for $5 \mathrm{~min}$. Then, excess regent was washed with distilled water for 
10min. The sections were discarded excess liquid and incubated with DAPI regent (Blue) for $10 \mathrm{~min}$ at room temperature. Finally, the sections were washed with PBS and then enclosed with antifade mounting medium.

\subsubsection{Western Blot}

After transplantation for $10 \mathrm{~d}$, grafts were separated, lysed and reacted with SDS loading buffer. Electrophoresis was performed and protein was transferred to PVDF membrane. The target membrane was then treated with blocking buffer and then covered with anti-IL-27Ra mAb solution and GAPDH solution overnight. Later, membrane was washed with TBST buffer and covered with HRP-labeled Goat Anti-Rat IgG solution and HRP-labeled Goat Anti-Rabbit IgG solution, respectively. Finally, membrane was washed with TBST buffer, following by ECL substrate covering. Band was scanned using Tanon 5200 imaging system scanner and analyzed with Image $\mathrm{J}$ software.

\subsection{Statistical analysis}

All data were quoted as mean \pm standard deviation (mean \pm SD) and each data point arised from 3 independent experiments. Comparisons between two groups were analyzed using the unpaired student's t-test. Correlation between DLU/ $\mathrm{mm}^{2}$ of ${ }^{125} \mathrm{I}-\mathrm{rL}-27$ and IL-27Ra expression was calculated by correlation assay. Statistically significant level was set at $P<0.05$.

\section{Results}

\subsection{Radiochemistry}

The labeling yields of ${ }^{125} \mathrm{I}$-rlL-27 and ${ }^{125}$ I-anti-IL-27Ra mAb were $84.4 \%$ and $99.0 \%$, respectively. The radiochemistry purity of these radio-probes was $93.3 \%$ and $95.3 \%$. The stabilities of ${ }^{125}$ I-rlL-27 and ${ }^{125}$ anti-IL-27Ra mAb were over $90 \%$ in saline and mouse serum even after $24 \mathrm{~h}$, respectively. The results showed that ${ }^{125} \mathrm{I}$-rlL-27 and ${ }^{125} \mathrm{I}$-anti-IL-27Ra mAb were quite stable. The $\log \mathrm{D}_{\mathrm{o} / \mathrm{w}}$ values for ${ }^{125} \mathrm{I}$-rlL-27 was $-1.18 \pm 0.23$, which means ${ }^{125} \mathrm{I}-\mathrm{rlL}-27$ possess hydrophilic character.

\subsection{Cell binding assays}

\subsubsection{Saturation}

Typical saturation graphs obtained after incubation of $1 \times 10^{6}$ cells with ${ }^{125}$ I-rlL-27 was shown in Figure $1 \mathrm{~A}-\mathrm{B}$. $B_{\max }$ values of ${ }^{125} \mathrm{I}$-rlL-27 on allo-reactive and syn-reactive splenocytes were $2545 \mathrm{cpm} / 10^{6}$ cells and $1607 \mathrm{cpm} / 10^{6}$ cells, respectively. Moreover, $K_{d}$ values were found $48.59 \mathrm{nM}$ and $49.04 \mathrm{nM}$ for allo- and syn-reactive splenocytes, respectively.

\subsubsection{Competition}


Figure $1 \mathrm{C}$ showed the binding of ${ }^{125} \mathrm{I}-\mathrm{rlL}-27$ decreased as anti-IL-27Ra mAb increased. Using the $\mathrm{K}_{\mathrm{d}}$ value of ${ }^{125} \mathrm{I}-\mathrm{rlL}-27$ from saturation assay, the determination the $\mathrm{K}_{\mathrm{i}}$ value was $769.9 \mathrm{nM}$ by Cheng-Prusoff equation.

\subsection{Small animal in vivo experiments}

\subsubsection{Blood clearance assay.}

To understand how fast ${ }^{125} \mathrm{I}$-rlL-27 cleared in vivo, blood clearance assay was performed. The blood clearance was represented as Clearance Value (CL). The allo-group displayed significantly lower concentration of ${ }^{125} \mathrm{I}$-rlL-27 than ${ }^{125} \mathrm{I}$-anti-IL-27Ra mAb in blood $(0.450 \pm 0.095 \mathrm{vs} 0.808 \pm 0.089 \mathrm{ng} / \mu \mathrm{L}$, $\mathrm{p}<0.01)$, and syn-group showed same pattern $(0.342 \pm 0.281 \mathrm{vs} 0.8967 \pm 0.0753 \mathrm{ng} / \mu \mathrm{L}, \mathrm{p}<0.05)$ at $24 \mathrm{~h}$ post injection. AUC (Area under curve) was shown in Figure 2, and the retention of ${ }^{125}{ }^{-r l L}-27$ in blood was shorter than that of ${ }^{125}$-anti-IL-27Ra mAb in allo-group ( $35.52 \mathrm{vs} 53.17 \mathrm{~h} \cdot \mathrm{ng} / \mu \mathrm{L}$ ) at $24 \mathrm{~h}$. The syn-group showed the shorter retention of ${ }^{125} \mathrm{I}$-rlL-27 than ${ }^{125} \mathrm{I}$-anti-IL-27Ra mAb (31.53vs $\left.52.65 \mathrm{~h} \cdot \mathrm{ng} / \mu \mathrm{L}\right)$ at $24 \mathrm{~h}$. ${ }^{125}$ I-rIL-27 demonstrated faster blood clearance compared with ${ }^{125}$ I-anti-IL-27Ra mAb in allo-group (13.20 vs $9.43 \mu \mathrm{L} / \mathrm{h}$ ) and syn-group ( 14.87 vs $9.53 \mu \mathrm{L} / \mathrm{h}$ ) at $24 \mathrm{~h}$. All these suggested a significantly faster blood clearance of ${ }^{125} \mathrm{I}$-rIL-27 than ${ }^{125} \mathrm{I}$-anti-IL-27Ra mAb in allograft rejection model mice.

\subsubsection{Dynamic whole-body phosphor-autoradiography imaging.}

To investigate ${ }^{125}$ I-rlL-27 imaging in vivo, we performed dynamic whole-body phosphor-autoradiography imaging. As shown in Figure 3A, high uptake of ${ }^{125} \mathrm{I}$-rlL-27 in allogeneic graft (DLU/mm²: $434241.58 \pm$ 53524.20) was obtained at $6 \mathrm{~h}$ after intravenous injection and then reached a plateau up to $24 \mathrm{~h}$ (DLU/mm²: $163603.46 \pm 56677.03)$, while lower uptake was obtained in syngeneic graft $\left(\mathrm{DLU} / \mathrm{mm}^{2}\right.$ : $66401.60 \pm 29698.30)(p<0.01)$. In vivo specificity of ${ }^{125}$ I-rIL-27 was confirmed by blocking studies using excess unlabeled anti-IL-27Ra mAb (DLU/mm²: $68252.033 \pm 38373.75)$. Ex vivo autoradiography showed apparently high activity accumulation in allograft. Similar result of ${ }^{125} \mathrm{I}$-anti-IL-27Ra mAb was observed at $48 \mathrm{~h}$, and the uptake of ${ }^{125} \mathrm{I}$-anti-IL-27Ra mAb in allogeneic graft was also higher than that in syngeneic graft (Figure 3B). However, imaging using ${ }^{125} \mathrm{I}-\mathrm{rLL}-27$ in allogeneic graft exhibited lower background in comparison with that using ${ }^{125} \mathrm{I}$-anti-IL-27Ra mAb. These indicated that ${ }^{125} \mathrm{I}$-rlL-27 could target allograft specifically and yield a better imaging with high contrast and low background.

\subsubsection{Biodistribution assay}

In order to have a first insight into the potential relevance of ${ }^{125} \mathrm{I}-$-rL -27 for transplantation imaging, biodistribution assay was performed using skin transplantation mice. The biodistribution data of ${ }^{125} \mathrm{I}$-rlL27 was shown in Figure 4A. Higher uptake was observed in allogeneic skin graft compared with that in syngeneic group (Figure 4B). Activity uptake of ${ }^{125} \mathrm{I}$-rlL-27 in allograft was higher than that in syngraft 
(\%ID/g: $5.648 \pm 1.735$ vs $1.751 \pm 0.967, p<0.01)$. T/NT ratio and T/B ratio significantly increased in allogroup compared with syn-group in Figure 4B-C.

More interestingly, compared with ${ }^{125} \mathrm{I}$-anti-IL-27Ra mAb, fewer ${ }^{125} \mathrm{I}-\mathrm{rIL}-27$ in blood was obtained at $24 \mathrm{~h}$ post injection not only in allo-group (\%ID/g: $6.960 \pm 0.754$ vs $4.083 \pm 0.710, p<0.01$ ), but also in syn-group (\%ID/g: $6.090 \pm 0.508$ vs $3.230 \pm 1.835, p<0.05$ ). Additionally, the activity uptake of ${ }^{125}$ I-rlL-27 was also lower than that of ${ }^{125} \mathrm{I}$-anti-IL-27Ra mAb in liver, lung, kidney, spleen. These indicated ${ }^{125} \mathrm{I}$-rIL-27 could specifically recognize IL-27Ra over-expressed in the allograft and have favorable imaging with low background.

\subsubsection{IL-27Ra expression in rejecting allograft.}

To study the correlation between activity accumulation of ${ }^{125} \mathrm{I}$-rIL-27 and IL-27Ra expression in rejecting allograft, IF staining was performed on day 10 post transplantation, so as to determine the IL-27Ra expression.

The HE staining in Figure 5A confirmed that severe rejection response was occurred in allogeneic graft, while mild inflammation in syngeneic graft. IL-27Ra expression was obviously higher in allograft (Figure $5 B)$. The activity accumulation ( $\mathrm{DLU} / \mathrm{mm}^{2}$ ) in graft had a positive correlation with IL-27Ra expression (Figure 5C). Fluorescence imaging also confirmed the higher IL-27Ra expression on the surface of infiltrated cells in rejecting allograft (Figure 5D). All these suggested that ${ }^{125}$-rIL-27 could specifically bind the IL-27Rain the allograft, monitoring the acute rejection.

\section{Discussion}

Early acute allorejection is usually more responsive to the therapy of allograft transplantation, and thus detection of acute rejection detection timely could benefit for the prognosis[37]. To data, molecular imaging with specific radio-probes was a promising method responsible for the detection of allograft rejection[22]. Recently, IL-27, a pleiotropic cytokine with pro-inflammation properties, was reported with enhanced antivirus and antitumor activities, and it participated in the rejection response[38][39][40][41]. IL-27 could promote infiltration of $\mathrm{CD} 4^{+} \mathrm{T}$ cell and $\mathrm{CD} 8^{+} \mathrm{T}$ cell in tumor, up-regulate IFN- $\gamma$, Granzyme $B$ and Perforin production, resulting in improved antitumor effect of T cell[10]. Moreover, IL-27 could also boost NK cell proliferation and cytotoxic activity synergistically with IL-15/IL-18[42]. All these indicated that IL27/IL-27R was a promising target in pro-inflammation immune response. IL-27Ra, the subunit of IL-27 receptor which is also expressed on the T cell and macrophage, had highest expression on the top acute rejection period in the allograft[43][44][45]. In our previous study, ${ }^{125}$ I-anti-IL-27Ra mAb has been found with high specificity towards IL-27Ra[18]. However, it had limitations in non-specific binding to Fc recognition, slow metabolism and clearance, as compared with small-sized antibody fragment or ligand[46][30]. Therefore, small-sized radio-probe could provide a better imaging with low background. 
Cytokine was a small-sized ligand of the cytokine receptor which is expressed on surface of effector cells[47]. Many radio-probes of cytokine have already been applied in targeting imaging[48][49][50][51]. Radiolabelled IL-2 probes were used in clinics for in targeted detection of the lymphocytic infiltration in transplantation and atherosclerotic plaque[48][49]. Glaudemans et al found symptomatic plaques with

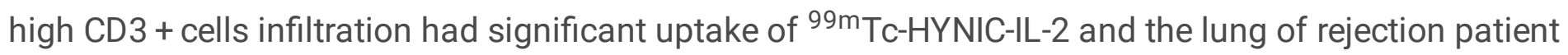
had increased ${ }^{99 m}$ Tc-HYNIC-IL-2 uptake. However, th their research, no side effect was found when administration of ${ }^{99 \mathrm{~m}}$ Tc-HYNIC-IL-2. We also developed the targeted radio-probe ${ }^{125}$ I-rIL-27 and also found no side effect in the mouse model.

In vitro experiment showed our ${ }^{125} \mathrm{I}-$-rL-27 had a specific binding to the IL-27Ra on the spleen cells. However, binding ability and affinity of ${ }^{125}$ I-rIL-27 was lower than that of ${ }^{125}$ I-anti-IL-27Ra mAb. This might be due to that ${ }^{125}$ I-anti-IL-27Ra mAb have non-specific Fc fragment binding. Matsushima et al developed ${ }^{125}$-labeled IL $1 \beta$ in a human large granular lymphocyte cell line (YT cells) and this radio-probe showed higher affinity of $0.1 \mathrm{nM}\left(\mathrm{K}_{d}\right.$ value) compared with our probe[52]. It maybe was due to the different receptor expression of the cells. Besides, the isolation process of spleen cells may also result in some loss of receptors[53].

In the imaging of [ ${ }^{124}$ I]I-F8-IL10, it was suggested that targeted area had highest uptake and target-tobackground ratios at $24 \mathrm{~h}$ post injection of the radio-probe[34]. Therefore, we carried out the biodistribution, blood clearance of ${ }^{125} \mathrm{I}-\mathrm{rl}$ - 27 within $24 \mathrm{~h}$ after radio-probe injection. In the blood clearance assay, ${ }^{125} \mathrm{I}$-rIL-27 showed faster blood clearance than ${ }^{125}$ I-anti-IL-27Ra, which was might due to the different cytokine and antibody glycosylation level, influencing the receptor recognition and blood clearance[54]. Blood clearance assay showed shorter retention of ${ }^{125} \mathrm{I}$-anti-IL-27Ra in blood compared with monoclonal antibody, which might be due to Fc recognition[55]. Whole-body phosphor-

autoradiography imaging demonstrated that the allograft had more activity accumulation than syngeneic graft, and this accumulation could be blocked by the excess of anti-IL-27Ra mAb. Lower background was also observed at $24 \mathrm{~h}$ in ${ }^{125} \mathrm{I}$-rlL-27 group compared with ${ }^{125} \mathrm{I}$-anti-IL-27Ra. Tumor necrosis factor superfamily (TNFSF) contains CD40L, FasL, TRAIL, LiGHT, VEGI, lymphotoxin alpha, lymphotoxin beta and lymphotoxin alpha1/beta2, which could be fused with F8 antibody for tumor targeting. in the biodistribution suggested that the \%ID/g of ${ }^{125} \mathrm{I}-\mathrm{rlL}-27$ in allograft was similar to that of F8-TRAILtrunc, lower than that of F8-CD40L and higher than that of other TNFSF in tumor[56]. The reason may be the different receptor expression and affinity of different cytokine to the receptors. The \%ID/g of ${ }^{125} \mathrm{I}-\mathrm{rIL}$ $27(47.8 \mathrm{KDa})$ in blood was higher than that of F8-TNFSF, F8-IL-10 (18.6 KDa), ${ }^{99 m} \mathrm{TC}^{-V E G F} \mathrm{~V}_{165}(16 \mathrm{KDa})$, which was probably due to the lower molecular weight of other cytokine[50]. However, the activity of ${ }^{125}$ rlL-27 in blood was much lower compared with that of ${ }^{125} \mathrm{I}$-anti-IL-27Ra $(155 \mathrm{KDa})$, perhaps caused by the non-specific Fc binding of ${ }^{125} \mathrm{I}$-anti-IL-27Ra. The uptake of ${ }^{125} \mathrm{I}$-rlL-27 in lung was higher than that in other organs except graft and blood. This might result from the enrichment of IL-27Ra-overexpressed immune cells in lung. Meanwhile, blood pollution should also be considered. The activity accumulation in kidney was found higher than liver, which may be because of the hydrophilic character of ${ }^{125} \mathrm{I}-\mathrm{rlL}-27$. Moreover, 
125I-rlL-27 accumulation was also found to have close correlation with IL-27Ra expression of the graft. All these indicated that ${ }^{125}$-rIL-27 was a promising radiotracer which could specifically target IL-27Ra for the imaging of acute rejecting allograft with faster blood clearance and low background, compared with ${ }^{125}$ anti-IL-27Ra mAb.

\section{Conclusion}

In this study, the acute allograft rejection could be detected by targeting IL-27Ra in allograft specifically with ${ }^{125} \mathrm{I}$-rlL-27. The rejecting allograft had higher specific ${ }^{125} \mathrm{I}-\mathrm{rlL}-27$ uptake than non-rejecting syngeneic graft and the activity accumulation was in close correlation with IL-27Ra expression of the graft. More importantly, low background and fast clearance was obtained for ${ }^{125} \mathrm{I}$-rlL-27 compared with ${ }^{125} \mathrm{I}$-anti-IL27Ra mAb. Imaging with this small-sized radio-probe might be a promising strategy for non-invasive monitoring of IL-27Ra-overexpressed rejecting allograft.

\section{Abbreviations}

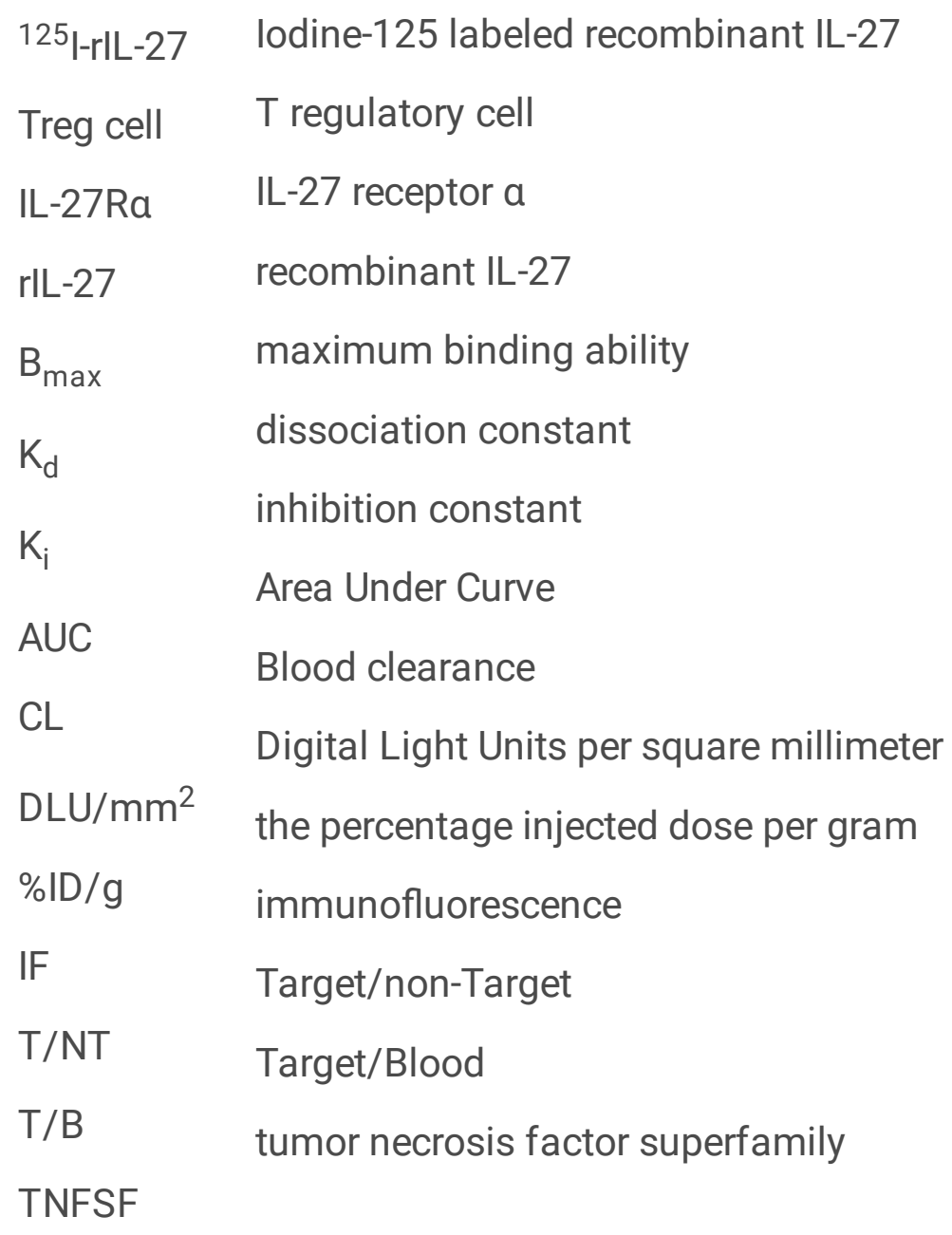

\section{Declarations}


Acknowledgments

This work gained great support by Core facilities sharing platform of Shandong University. This research was funded by grants from the National Natural Science Foundation of China (81371601) and Natural Science Foundation of Shandong Province (ZR2019MH019).

\section{Conflicts of interest}

The authors have no conflicts of interest to disclose.

\section{References}

1. Grenda R, Kaliciński P. Combined and sequential liver-kidney transplantation in children. Pediatr Nephrol (Berlin Germany). 2018;33(12):2227-37.

2. Jalalzadeh $\mathrm{M}$, et al. The impact of acute rejection in kidney transplantation on long-term allograft and patient outcome. Nephro-urology monthly. 2015;7(1):e24439-9.

3. Tsai M-K, et al. Decreased Acute Rejection and Improved Renal Allograft Survival Using Sirolimus and Low-Dose Calcineurin Inhibitors without Induction Therapy. The International Journal of Artificial Organs. 2009;32(6):371-80.

4. Peshkova IO, et al. IL-27 receptor-regulated stress myelopoiesis drives abdominal aortic aneurysm development. Nature communications. 2019;10(1):5046-6.

5. Yao G, et al. Mesenchymal stem cell transplantation alleviates experimental Sjögren's syndrome through IFN- $\beta /$ IL-27 signaling axis. Theranostics. 2019;9(26):8253-65.

6. Huang Z, et al. IL-27 promotes the expansion of self-renewing CD8(+) T cells in persistent viral infection. J Exp Med. 2019;216(8):1791-808.

7. Bastian D, et al. IL-27 Receptor Signaling on T cells Augments GVHD Severity through Enhancing Th1 Responses. J Immunol Res Ther. 2018;3(1):151-7.

8. Wang Q, et al. Tristetraprolin inhibits macrophage IL-27-induced activation of antitumour cytotoxic T cell responses. Nature communications. 2017;8(1):867-7.

9. Jones GW, et al. IL-27: a double agent in the IL-6 family. Clin Exp Immunol. 2018;193(1):37-46.

10. Zhu J, et al. IL-27 gene therapy induces depletion of Tregs and enhances the efficacy of cancer immunotherapy. JCl insight. 2018;3(7):e98745.

11. Petes $C$, et al. IL-27 enhances LPS-induced IL-1 $\beta$ in human monocytes and murine macrophages. J Leukoc Biol. 2017;102(1):83-94.

12. Kumar P, et al. IL-27 promotes NK cell effector functions via Maf-Nrf2 pathway during influenza infection. Scientific reports. 2019;9(1):4984-4.

13. Benzimra M, Calligaro GL, Glanville AR. Acute rejection. Journal of thoracic disease. 2017;9(12):5440-57. 
14. Gaignage $M$, et al. The TLR7 ligand R848 prevents mouse graft-versus-host disease and cooperates with anti-interleukin-27 antibody for maximal protection and regulatory T-cell upregulation. Haematologica. 2019;104(2):392-402.

15. Petes C, et al. Interleukin (IL)-6 Inhibits IL-27- and IL-30-Mediated Inflammatory Responses in Human Monocytes. Front Immunol. 2018;9:256.

16. Qiu S-L, et al. Cigarette Smoke Induction of Interleukin-27/WSX-1 Regulates the Differentiation of Th1 and Th17 Cells in a Smoking Mouse Model of Emphysema. Frontiers in immunology. 2016;7:553-3.

17. Xu J, et al. Alternatively Expressed Genes Identified in the CD4 + T Cells of Allograft Rejection Mice. Cell Transplant. 2011;20(2):333-50.

18. Zhao S, et al. IL-27Ra: A Novel Molecular Imaging Marker for Allograft Rejection. Int J Mol Sci. 2020;21(4):E1315.

19. Sharfuddin and A.J.C.J.o.t.A.S.o.N. Cjasn. Renal Relevant Radiology: Imaging in Kidney Transplantation. 9(2): p. 416-429.

20. Roden A, et al., Diagnosis of Acute Cellular Rejection and Antibody-Mediated Rejection on Lung Transplant Biopsies: A Perspective From Members of the Pulmonary Pathology Society. Archives of Pathology \& Laboratory Medicine, 2016. 141.

21. Huang $Y$, et al. Measurement and comparison of $T 1$ relaxation times in native and transplanted kidney cortex and medulla. Journal of magnetic resonance imaging: JMRI. 2011;33(5):1241-7.

22. Köhnke R, et al. Update on imaging-based diagnosis of acute renal allograft rejection. American Journal of Nuclear Medicine Molecular Imaging. 2019;9:110-26.

23. Liao $\mathrm{T}$, et al., Noninvasive quantification of intrarenal allograft C4d deposition with targeted ultrasound imaging. 2019.

24. Xu DM, et al. Risk Factors for Severe Bleeding Complications in Percutaneous Renal Biopsy. Am J Med Sci. 2017;353(3):230-5.

25. $\mathrm{Chi} \mathrm{H}$, et al. Multiple biopsy passes and the risk of complications of percutaneous liver biopsy. European Journal of Gastroenterology Hepatology. 2017;29(1):36-41.

26. Freise AC, et al., ImmunoPET Imaging of Murine CD4 + T Cells Using Anti-CD4 Cys-Diabody: Effects of Protein Dose on T Cell Function and Imaging.

27. Mall S, et al., Immuno-PET Imaging of Engineered Human T Cells in Tumors. 2016. 76(14): p. 4113.

28. Sun $\mathrm{H}$, et al. Non-invasive imaging of allogeneic transplanted skin graft by 131 l-anti-TLR5 mAb. J Cell Mol Med. 2014;18(12):2437-44.

29. Berraondo $P$, et al. Cytokines in clinical cancer immunotherapy. Br J Cancer. 2019;120(1):6-15.

30. Ehlerding EB, et al. Antibody and fragment-based PET imaging of CTLA-4 + T-cells in humanized mouse models. Am J Cancer Res. 2019;9(1):53-63.

31. Ying T, et al. Monomeric IgG1 Fc molecules displaying unique Fc receptor interactions that are exploitable to treat inflammation-mediated diseases. mAbs. 2014;6(5):1201-10. 
32. Hartimath SV, et al. Noninvasive monitoring of cancer therapy induced activated T cells using [18F]FB-IL-2 PET imaging. Oncolmmunology. 2017;6(1):e1248014.

33. Di Gialleonardo V, et al. N-(4-18F-fluorobenzoyl)interleukin-2 for PET of human-activated T lymphocytes. J Nucl Med. 2012;53(5):679-86.

34. Bruijnen STG, et al. F8-IL10: A New Potential Antirheumatic Drug Evaluated by a PET-Guided Translational Approach. Mol Pharm. 2019;16(1):273-81.

35. Shi D, et al. TLR5 is a new reporter for triple-negative breast cancer indicated by radioimmunoimaging and fluorescent staining. J Cell Mol Med. 2019;23(12):8305-13.

36. Zhang R. Donor-Specific Antibodies in Kidney Transplant Recipients. Clinical journal of the American Society of Nephrology: CJASN. 2018;13(1):182-92.

37. Belle L, et al. Blockade of interleukin-27 signaling reduces GVHD in mice by augmenting Treg reconstitution and stabilizing Foxp3 expression. Blood. 2016;128(16):2068-82.

38. Jung J-Y, Roberts LL, Robinson CM. The presence of interleukin-27 during monocyte-derived dendritic cell differentiation promotes improved antigen processing and stimulation of T cells. Immunology. 2015;144(4):649-60.

39. Petes $\mathrm{C}$, et al. The effects of CD14 and IL-27 on induction of endotoxin tolerance in human monocytes and macrophages. J Biol Chem. 2018;293(45):17631-45.

40. Wang Q, et al. Tristetraprolin inhibits macrophage IL-27-induced activation of antitumour cytotoxic T cell responses. Nat Commun. 2017;8(1):867.

41. Choi YH, et al. IL-27 enhances IL-15/IL-18-mediated activation of human natural killer cells. J immunother Cancer. 2019;7(1):168-8.

42. Iwasaki Y, et al. Interleukin-27 in T cell immunity. Int J Mol Sci. 2015;16(2):2851-63.

43. Holz K, et al. Differing Outcome of Experimental Autoimmune Encephalitis in Macrophage/Neutrophil- and T Cell-Specific gp130-Deficient Mice. Front Immunol. 2018;9:836.

44. Zhao $\mathrm{S}$, et al., IL-27 Ra(+) cells promoted allorejection via enhancing STAT1/3/5 phosphorylation. J Cell Mol Med, 2020.

45. Calzada V, et al. Fab(nimotuzumab)-HYNIC-99mTc: Antibody Fragmentation for Molecular Imaging Agents. Anticancer Agents Med Chem. 2016;16(9):1184-9.

46. Oppenheim JJ. The Future of the Cytokine Discipline. Cold Spring Harb Perspect Biol, 2018. 10(9).

47. Glaudemans A, et al., In vivo and in vitro evidence that Tc-99m-HYNIC-interleukin-2 is able to detect T lymphocytes in vulnerable atherosclerotic plaques of the carotid artery. European journal of nuclear medicine and molecular imaging, 2014. 41.

48. Telenga ED, et al. (99 m)Tc-HYNIC-IL-2 scintigraphy to detect acute rejection in lung transplantation patients: a proof-of-concept study. EJNMMI Res. 2019;9(1):41.

49. Galli F, et al. Radiolabeling of VEGF165 with $99 \mathrm{mTc}$ to evaluate VEGFR expression in tumor angiogenesis. Int J Oncol. 2017;50(6):2171-9. 
50. Gratz S, et al. Rapid imaging of experimental colitis with $(99 \mathrm{~m})$ Tc-interleukin-8 in rabbits. J Nucl Med. 2001;42(6):917-23.

51. Matsushima K, et al. Down-regulation of interleukin 1 (IL 1) receptor expression by IL 1 and fate of internalized 125I-labeled IL 1 beta in a human large granular lymphocyte cell line. J Immunol. 1986;137(10):3183-8.

52. Nieto JC, et al. Selective loss of chemokine receptor expression on leukocytes after cell isolation. PLoS One. 2012;7(3):e31297.

53. Ohtsubo K, Marth JD. Glycosylation in cellular mechanisms of health and disease. Cell. 2006;126(5):855-67.

54. Matera MG, et al. Monoclonal antibodies for severe asthma: Pharmacokinetic profiles. Respir Med. 2019;153:3-13.

55. Hemmerle T, et al. Tumor targeting properties of antibody fusion proteins based on different members of the murine tumor necrosis superfamily. J Biotechnol. 2014;172:73-6.

\section{Figures}

A

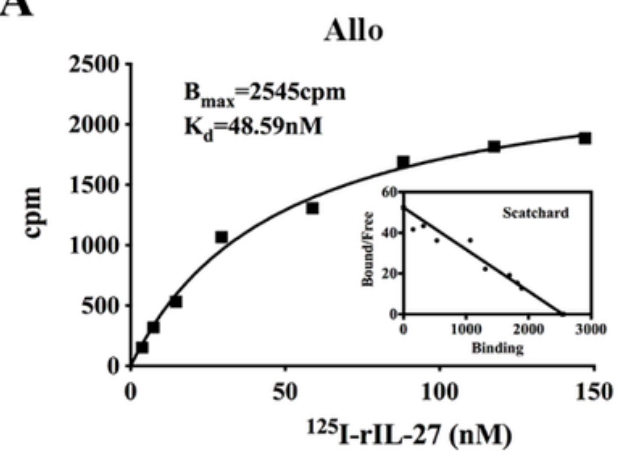

B

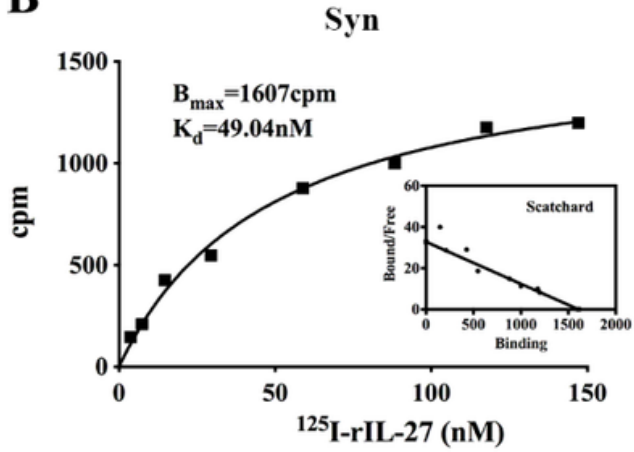

C

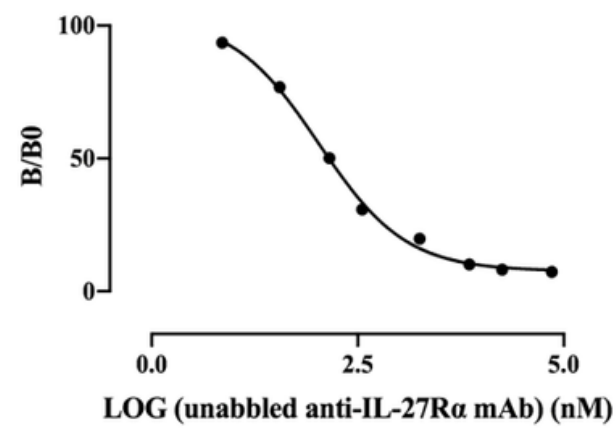

\section{Figure 1}

Binding assay of 125I-rlL-27 in vitro. Allogeneic and syngeneic skin transplantation mice were established and represented as Allo and Syn group. Spleen cells were isolated on day 10 post transplantation and competition binding assay was performed. A-B. The Saturation assay of alloreactive spleen cells (A) and synreactive spleen cells (B). C. The competitive binding of 125I-rlL-27 in alloreactive spleen cells. 


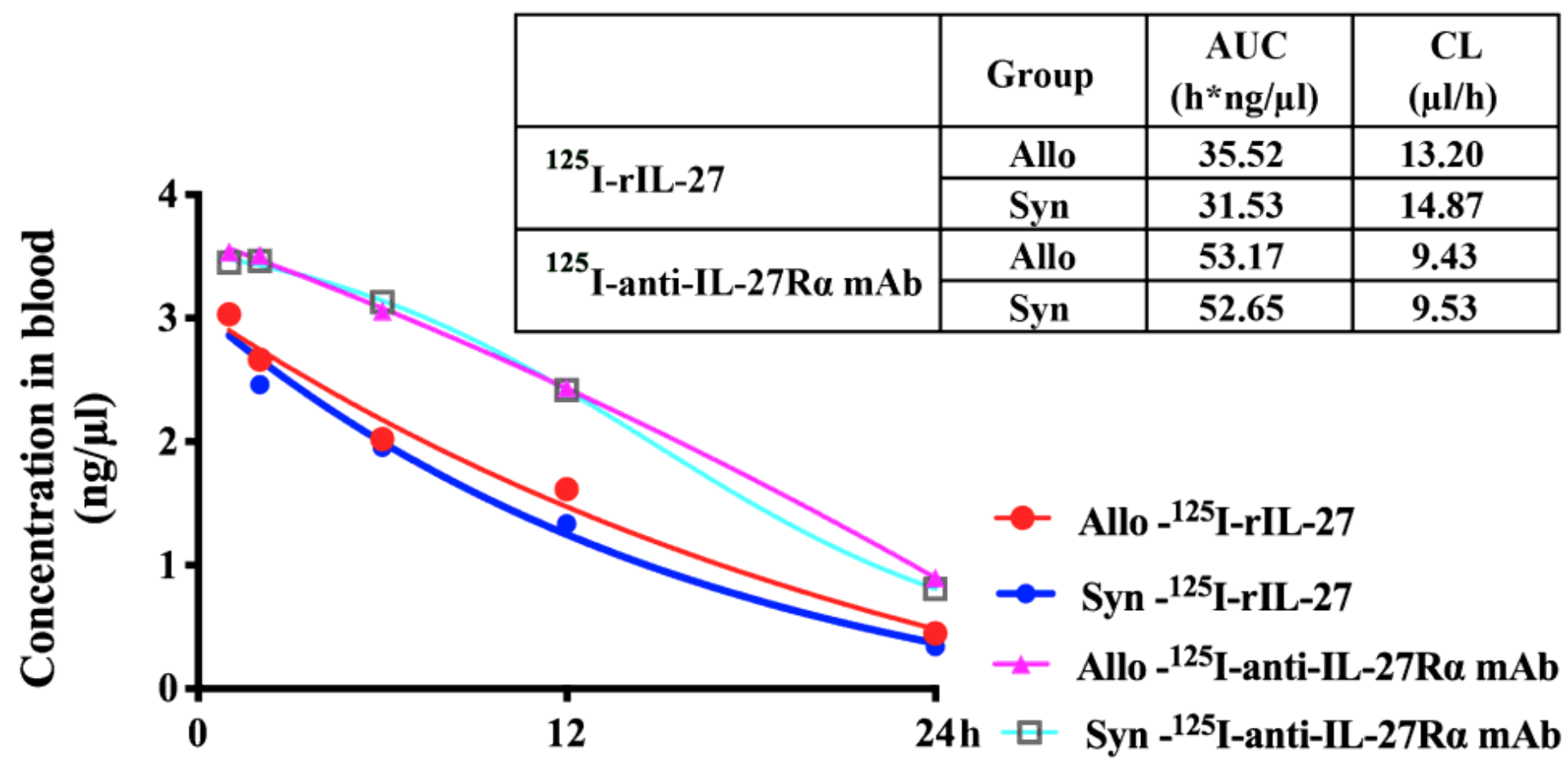

Figure 2

Blood clearance assay. Time- Radio probe concentration in blood curves after administration of 125I-IL27 and 125I-anti-IL-27Ra mAb in allogeneic and syngeneic transplantation mice. The inset table was the AUC from 1-24h of 125I-rIL-27 and 125I-anti-IL-27Ra mAb and blood clearance assay. 
A

Allo
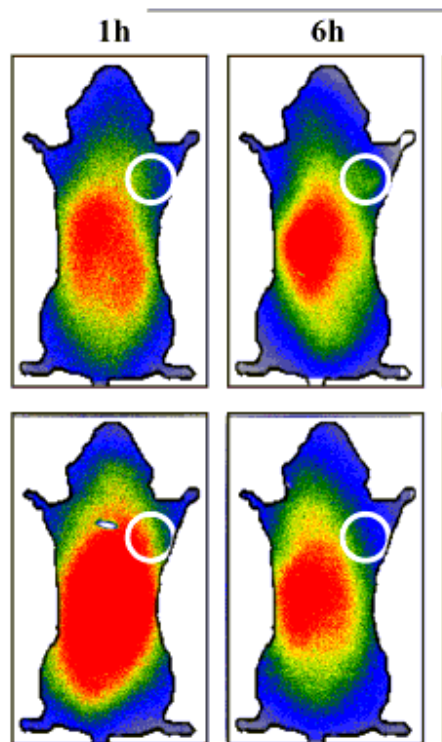

Syn

Allo-

Blocking
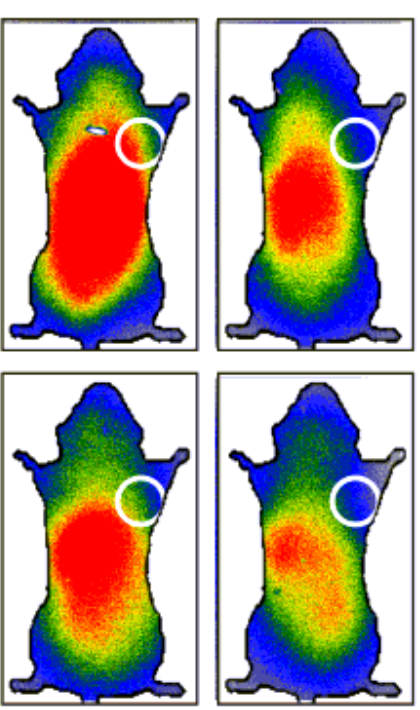
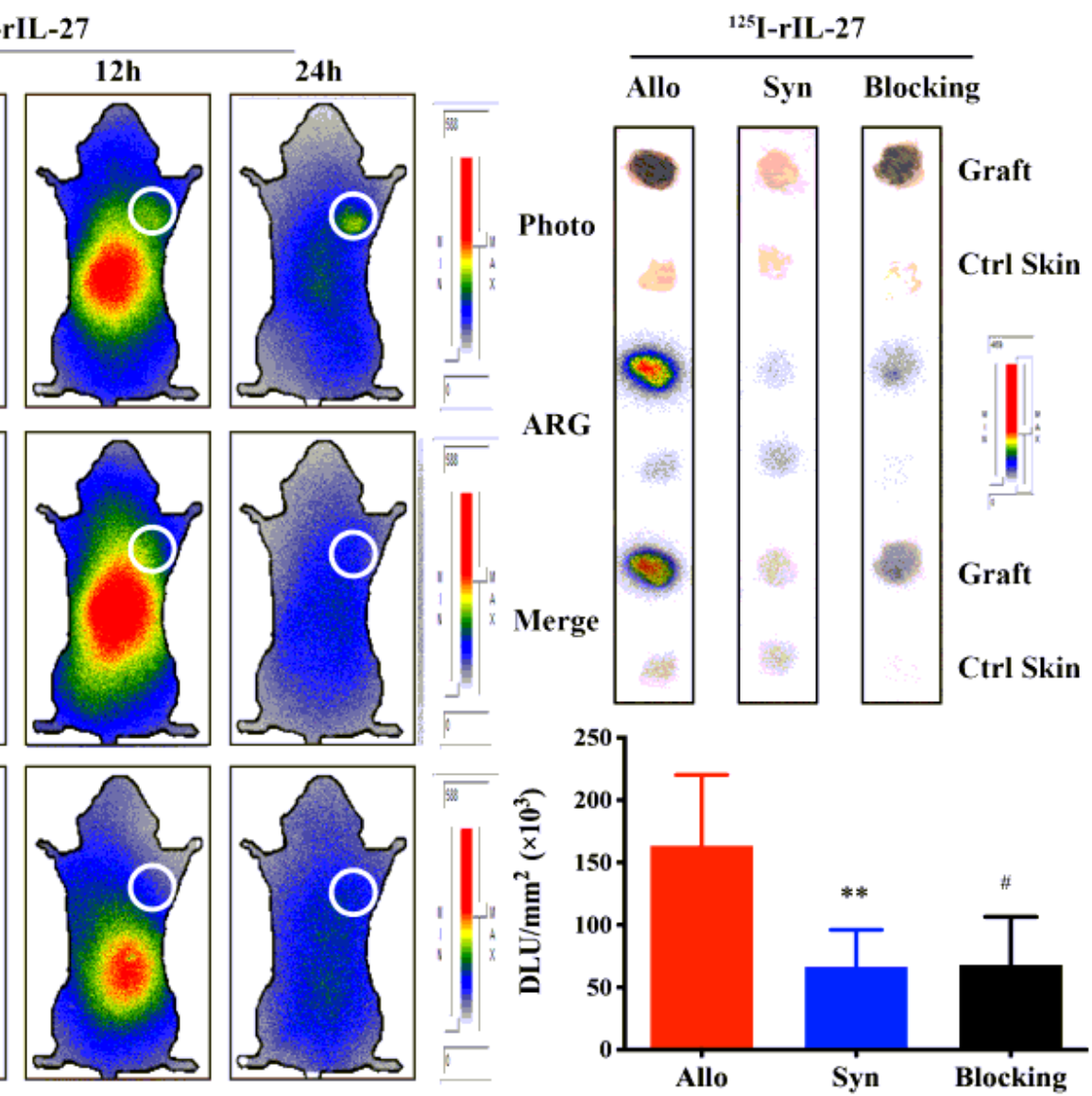

B

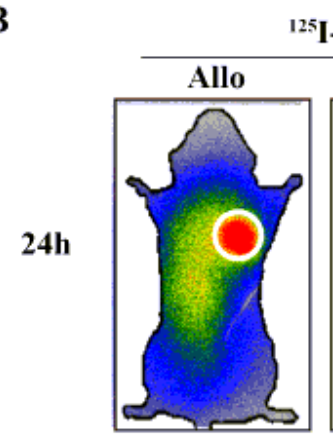

${ }^{5}$ I-anti-IL-27Ra mAb
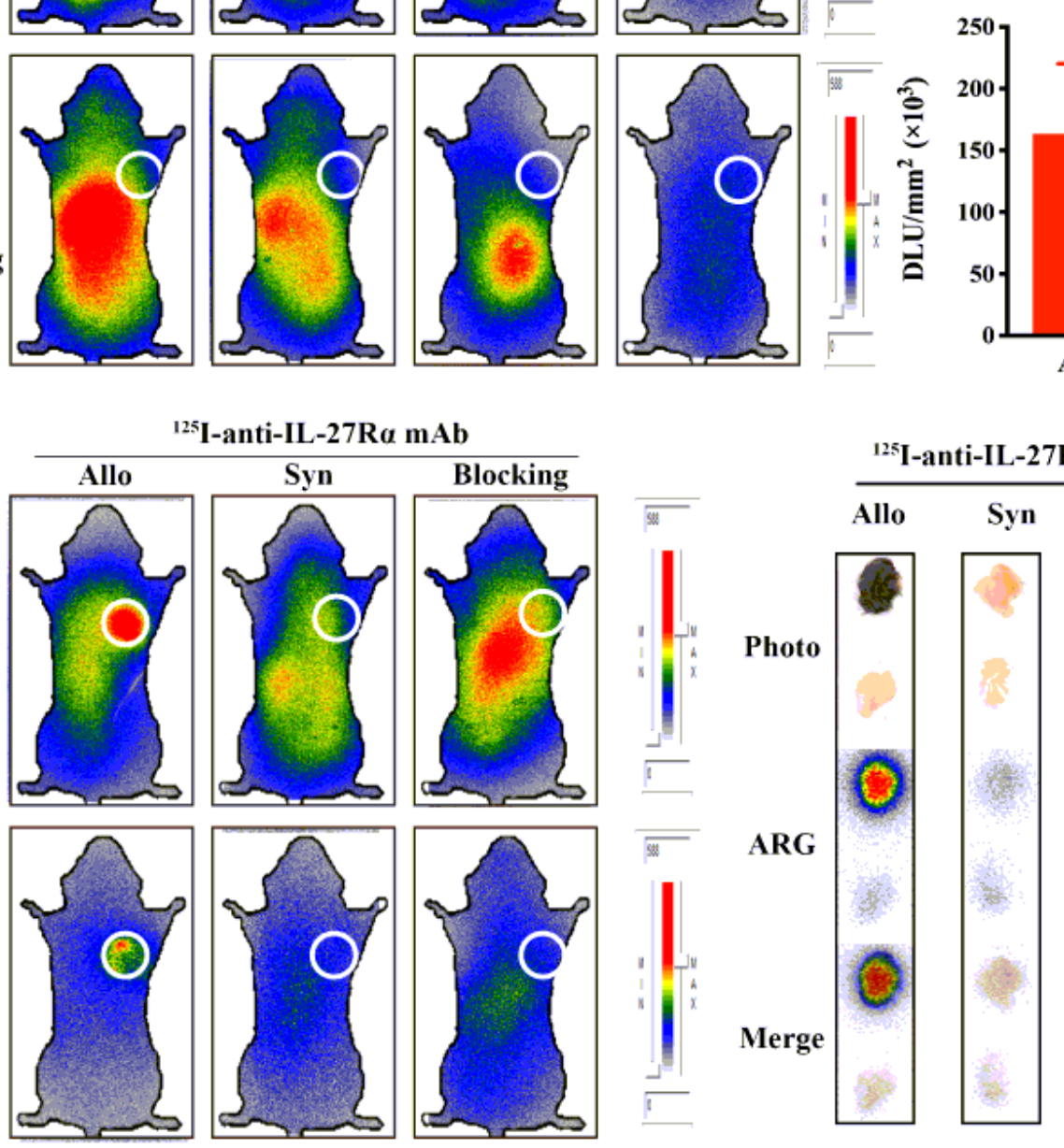

\section{Figure 3}

Dynamic whole-body phosphor-autoradiography imaging assay. Allogeneic and syngeneic skin transplantation mice were established and represented as Allo and Syn group. Mice was injected with 125I-rIL-27 and 125I-anti-IL-27Ra mAb on day 9 post transplantation and scanned at different time. Graft and opposite control skin were isolated on day 10 (24h after radio probe injection). ARG means the autoradiography. A. Imaging at 1, 6, 12, 24h post 125I-rIL-27 injection and DLU/mm2 assay. The circle 
indicated the position of the graft. B. Imaging at $24,48 \mathrm{~h}$ post $125 \mathrm{I}$-anti-IL-27Ra mAb injection. ${ }^{\star *} \mathrm{p}<0.01$ was used in Allo vs Syn group. \#p 0.01 was used in Allo vs Blocking group.

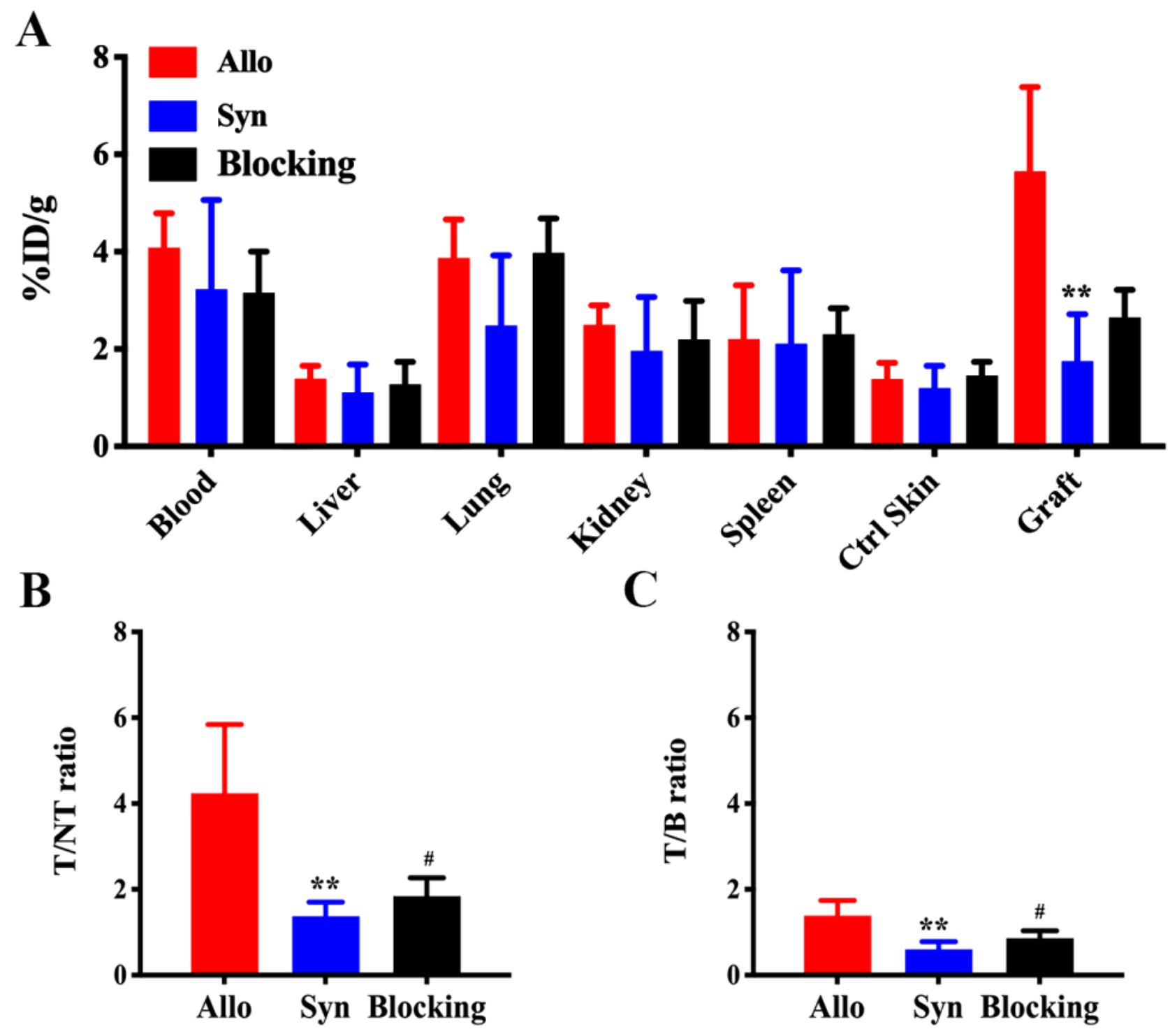

Figure 4

Bio-distribution study on day 10 post transplantation. The organ was separated from allografted and syngrafted mouse model on day 10 and biodistribution assay at $24 \mathrm{~h}$ post radio probe injection. $\mathrm{T} / \mathrm{NT}$ and T/B assay was calculated. A. Biodistribution assay of 125I-rlL-27 injection. B-C. T/NT ratio (B) and T/B ratio (C) by $125 \mathrm{I}$-rlL-27 injection. ${ }^{* *} \mathrm{p}<0.01$ was used in Allo vs Syn group. $\# p<0.05$ was used in Allo vs Blocking group. 
A

Allo

Syn
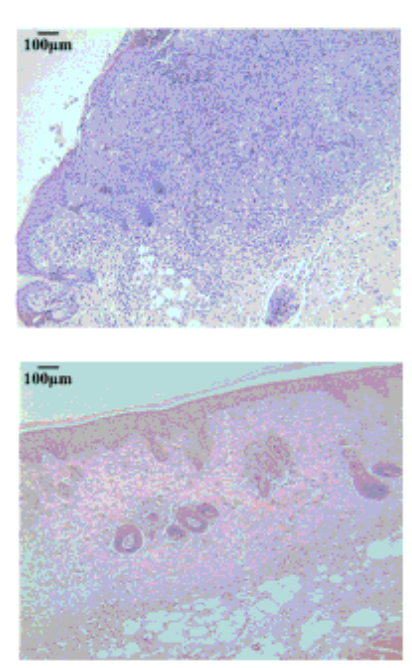

D

Allo
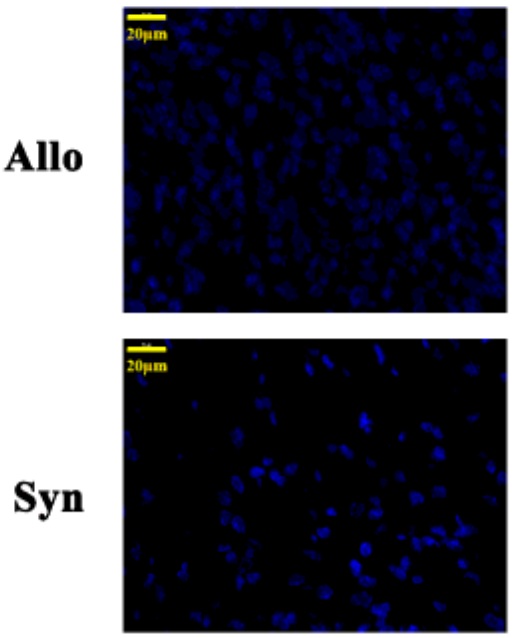

B
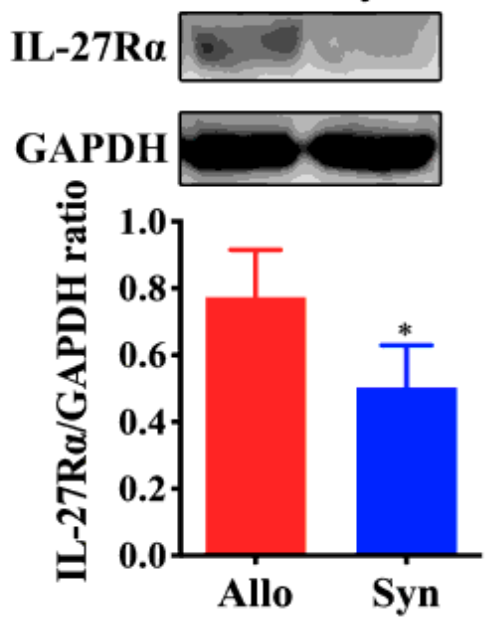

IL-27R $\alpha$.
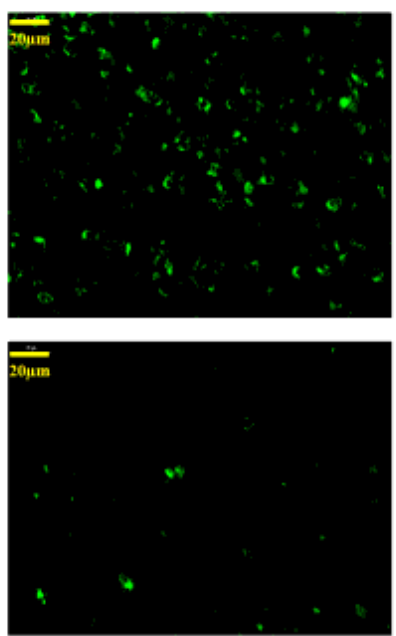

C

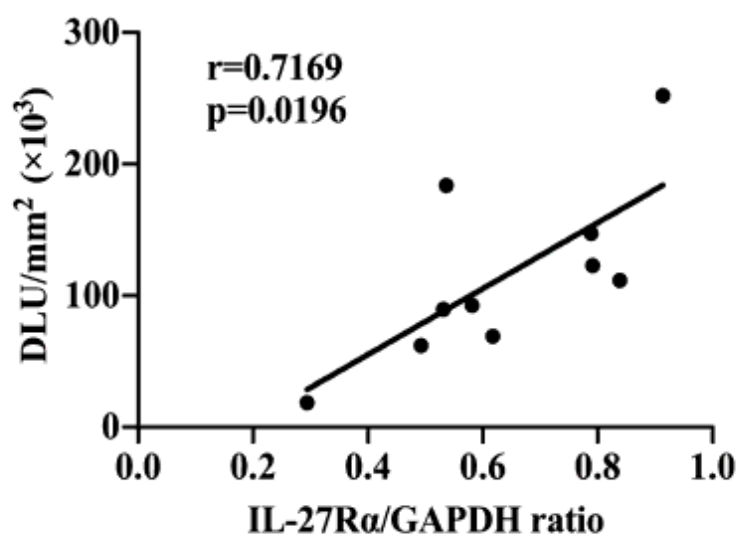

Merge
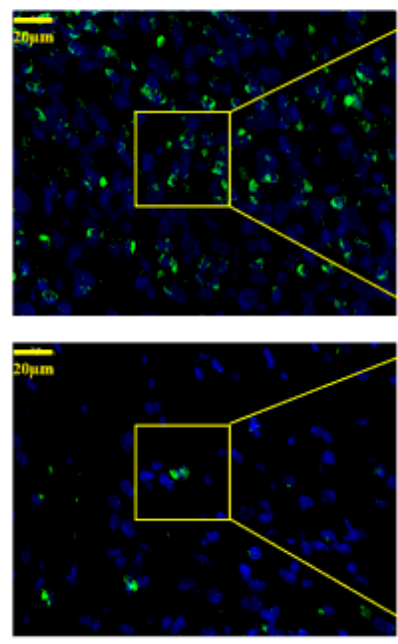

Enlarge
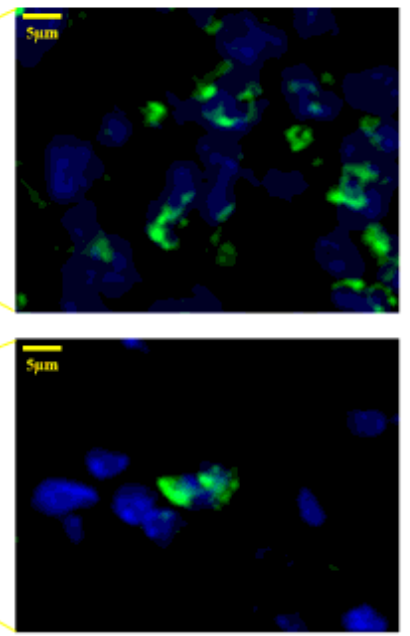

\section{Figure 5}

H\&E staining and IL-27Ra expression. The graft was isolated on day 10 post transplantation. A. H\&E staining of allogeneic and syngeneic graft. B. IL-27Ra expression was detected by Western blot assay. C. Correlation assay between 125I-rlL-27 accumulation and IL-27Ra expression in graft. D.IL-27Ra expression was detected by immunofluorescence staining. 